\title{
Novel technique for the extraction of ionization profiles from spatial density measurements
}

Cite as: Review of Scientific Instruments 72, 4362 (2001); https://doi.org/10.1063/1.1412856

Submitted: 21 February 2001 • Accepted: 29 August 2001 • Published Online: 27 November 2001

C. M. Deegan, C. E. Markham, M. Turner, et al.
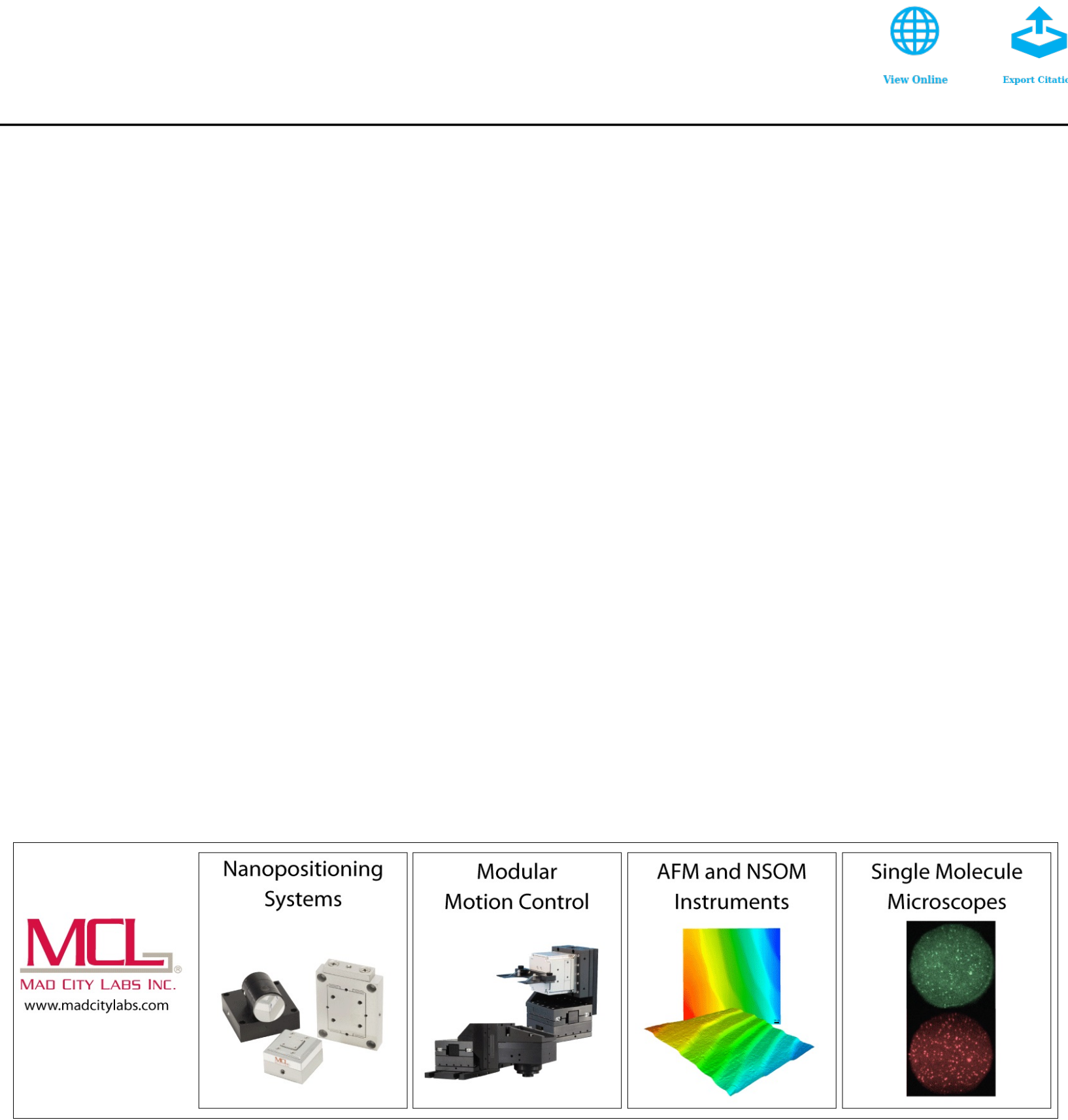


\title{
Novel technique for the extraction of ionization profiles from spatial density measurements
}

\author{
C. M. Deegan ${ }^{a)}$ \\ School of Informatics and Engineering, Institute of Technology Blanchardstown, Dublin 15, Ireland \\ C. E. Markham ${ }^{\text {b) }}$ \\ Department of Computer Science, National University of Ireland, Maynooth, Co. Kildare, Ireland \\ M. Turner and D. Vender \\ Plasma Research Laboratory, School of Physical Sciences, Dublin City University, Dublin 9, Ireland
}

(Received 21 February 2001; accepted for publication 29 August 2001)

\begin{abstract}
A method has been devised to extract the two-dimensional ionization profiles from spatially resolved ion density measurements made in a capacitively coupled argon rf plasma. This technique is valid if the production process is ionization and the loss process is ambipolar diffusion. As this procedure calculates the total production (ionization) term, processes such as two-step ionization and photoionization are included. The main benefit of this new technique is that it extracts ionization rates that pertain to the total ionization in the discharge. It follows that this technique could be used to determine when other ionization processes (e.g., photo- and two-step ionization) are important. On testing with experimental data, the inversion process was observed to reproduce the original source term, thereby verifying that the iteration process converges to a real solution. The calculated ionization rate using the integral method is in good agreement with the ionization profiles obtained from the density measurements. Hence, there is now a method of determining the ionization rate that is independent of electron energy probability function measurement and that includes all ionization processes, not just ground state ionization by electron-neutral collisions. (C) 2001 American Institute of Physics. [DOI: 10.1063/1.1412856]
\end{abstract}

\section{INTRODUCTION}

Low pressure, nonequilibrium weakly ionized gas discharge plasmas are used for a variety of materials processing applications. The most extensive applications are in microelectronics manufacturing, where plasma sputtering, etching, stripping, cleaning, and film deposition play key roles in this growing industry. ${ }^{1-3}$ Low-pressure, capacitively coupled rf glow discharges are still the most commonly used in industry for semiconductor fabrication. At low pressures, both the charged-particle concentration and spatial distribution are very important characteristics used in manufacturing, especially when considering spatial uniformity. A quantitative understanding of the spatial structure of processing discharges under processing conditions is therefore useful, especially when considering the discharge geometry. Knowledge of the ionization or source distribution is fundamental to the understanding of the spatial density distribution. Hence, the value of the technique is presented here.

Kitajima and co-workers, ${ }^{4}$ among many others have measured spatially resolved excitation profiles in capacitively coupled discharges in argon at low pressures. The main advantage of optical emission is that it may be time resolved to small fractions of the rf cycle. A disadvantage of optical emission studies is that actual ionization profiles are

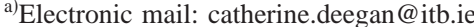

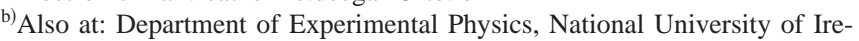
land, Maynooth, Co. Kildare, Ireland.
}

not measured, the ionization profile may only be considered as being similar to the excitation profile if there are similarities in the cross sections for the two processes. Even if both cross sections are similar, it is uncertain that the ionization profile could be quantitatively inferred from optical emission data as the electron energy probability function (EEPF) is also significant when calculating the ionization source term and optical emission gives no information on the EEPF.

The method described in this article has been devised to extract the total ionization profile in two dimensions from density measurements in a low-pressure argon discharge. The relevant transport equation is iteratively inverted to produce the ionization source term. This inversion technique will work whenever the transport equation is known, so extra effects that may contribute to ionization may be included. The sample case presented in this article is a simple case that proves the basic principles of the technique. It is valid if the production process is ionization and the loss process is ambipolar diffusion. It should also be clear that this procedure will only be valid where the ionization frequency is a great deal lower than the ion collision frequency so the diffusion assumption is valid. As this procedure calculates the total production (ionization) term, processes such as two-step ionization and photoionization are included.

\section{EXPERIMENTAL TECHNIQUE}

Argon plasmas were generated in a $240 \mathrm{~mm}$ diameter, cylindrical stainless-steel chamber with a powered electrode 
of diameter $143 \mathrm{~mm}$. The gap width may be varied from 2 to $9 \mathrm{~cm}$. Spatially resolved density measurements were made using a Langmuir probe. The operation of this probe has been described in previous publications. ${ }^{5,6}$ It was connected to the chamber via a Conflat ${ }^{\mathrm{TM}}$ flange and mounted on a bellows assembly, which allowed movement of the probe in both the axial $(z)$ and radial $(r)$ directions. Axial measurements were restricted by the probe port so most spatially resolved measurements were performed in gaps of $55 \mathrm{~mm}$ or less to allow the probe to take measurements near to the powered electrode. Charged particle density and EEPF measurements were made parallel and perpendicular to the discharge electrodes.

\section{THE INVERSION TECHNIQUE}

Using the simplifying assumptions of electron impact ionization and ambipolar diffusion to the walls, the ionization profile is determined from the density profiles using a purpose written " $\mathrm{C}$ " program. One axial profile, taken along the middle of the discharge axis, plus three to six radial profiles, taken at either end and along the midplane, were interpolated to form a two-dimensional $50 \times 50$ grid that represented the experimental density profile. The more data obtained, the more accurate the density profile. However, it was found that once data were obtained in the regions of interest, e.g., in areas where the density is at a maximum and/or changing significantly, then the technique would work well with otherwise sparse data.

The algorithm uses an iterative technique that works as follows:

(i) A "first guess" of the ionization profile is inserted into a $50 \times 50$ data array. This may simply be a flat distribution or the same as the density profile times some constant.

(ii) A density profile corresponding to the ionization profile in part (i) is obtained as follows. A time stepping algorithm is used to solve the particle transport equation, the diffusive flux;

$$
\Gamma=-D_{A} \nabla n,
$$

where $\Gamma$ is the particle flux, $n$ is the particle density, and $D_{A}$ is the diffusion coefficient. For nonequilibrium plasmas, where the electron temperature is much higher than the ion temperature, Eq. (2) gives an expression for the ambipolar diffusion coefficient, $D_{A}$;

$$
D_{A}=\mu_{i} T_{e},
$$

where $\mu_{i}$ is the ion mobility and $T_{e}$ is the electron temperature in $\mathrm{eV}$. The ion mobility was taken from data in Ref. 7 and the electron temperature from Langmuir probe measurements at the discharge center.

This continues until equilibrium is reached, i.e., no further change is measured in the density profile. This process yields a modeled density profile.

(iii) This modeled density profile is compared (point for point) with the experimental density profile. Should a difference be measured, the following correction is made on each point,

$$
G_{\text {new }}=G_{\text {old }} \frac{N_{\text {experiment }}}{N_{\text {model }}},
$$

where $G$ is the ionization rate and $N$ is the density value at each point. This technique is commonly known in spectroscopy as Gold's ratio method ${ }^{8}$ and is similar to the multiplicative algebraic reconstruction technique used in computed tomography. ${ }^{9}$

(iv) This technique can amplify noise in the density profile so nine-point neighborhood averaging is employed every few iterations.

(v) Steps (ii) to (iv) are repeated until the modeled density profile converges on the experimental density profile. The ionization profile is then saved to a file.

\section{RESULTS}

Figure 1(a) shows the experimental density profile for a 300 mTorr, $5 \mathrm{~W}$ argon plasma. Figure 1(b) shows the corresponding modeled density profile obtained on convergence. Figure 1(c) shows the two-dimensional ionization profile produced by the iteration process. It is clear from Figs. 1(a) and 1(b) that the algorithm can accurately reproduce the experimental density profile. Reducing the dataset to one-ninth of its original size resulted in a relative error of less than a factor of two over the entire profile. In fact, the relative error in the main region of interest, close to the powered electrode, was less than $50 \%$. This indicates that this technique works well with sparse data. However, it is recommended that data should always be collected from the areas of interest as mentioned before.

\section{CALCULATION OF THE IONIZATION RATE USING THE EEPF}

As the measured EEPF in a low-pressure argon discharge is rarely Maxwellian, one way of estimating the ionization rate constant is to weigh the ionization cross section with the electron distribution function and to numerically integrate the result. The average electron energy in argon plasmas is of the order of $3 \mathrm{eV}$ but the electrons do have a distribution of energies. The ionization threshold of argon is $15.76 \mathrm{eV}$ so only the tail of the distribution function and the cross section values near threshold contribute to the rate constant for ionization.

The Thomson cross section for ionization is given by ${ }^{9}$

$$
\sigma_{i z}=\pi\left(\frac{e}{4 \pi \varepsilon_{0}}\right)^{2} \frac{1}{\varepsilon}\left(\frac{1}{\varepsilon_{i z}}-\frac{1}{\varepsilon}\right) \varepsilon>\varepsilon_{i z},
$$

where $\sigma_{i z}$ is the cross section and $\varepsilon_{i z}$ is the ionization threshold energy. This expression is an approximation to experimentally determined cross sections ${ }^{10}$ but it reasonably follows the experimental cross section for argon, $\varepsilon_{i z}$ $=15.76 \mathrm{eV}$. If the electron distribution is assumed to be Maxwellian, then the rate constant $K_{i z}$ is

$$
\begin{aligned}
K_{i z} & =\left\langle\sigma_{i z}(v) v\right\rangle_{v} \\
& =\left(\frac{m}{2 \pi k T_{e}}\right)^{3 / 2} \int_{0}^{\infty} \sigma(v) v \exp \left(\frac{-m v^{2}}{2 k T_{e}}\right) 4 \pi v^{2} d v .
\end{aligned}
$$


(a)

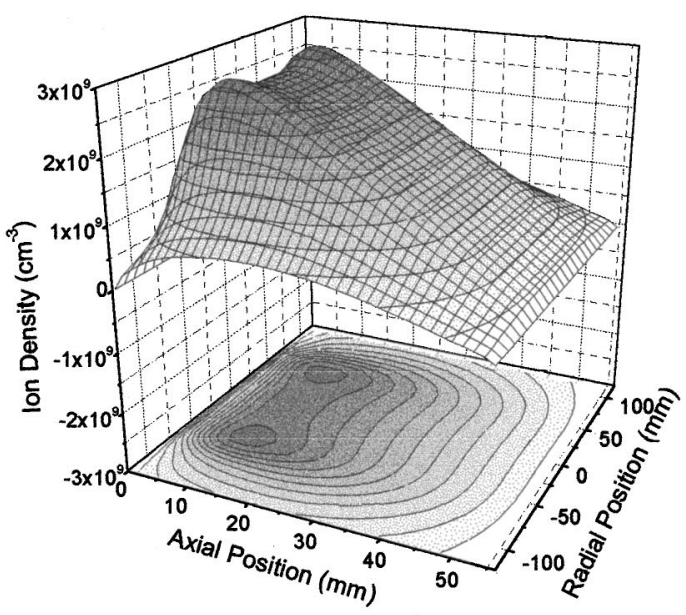

(b)

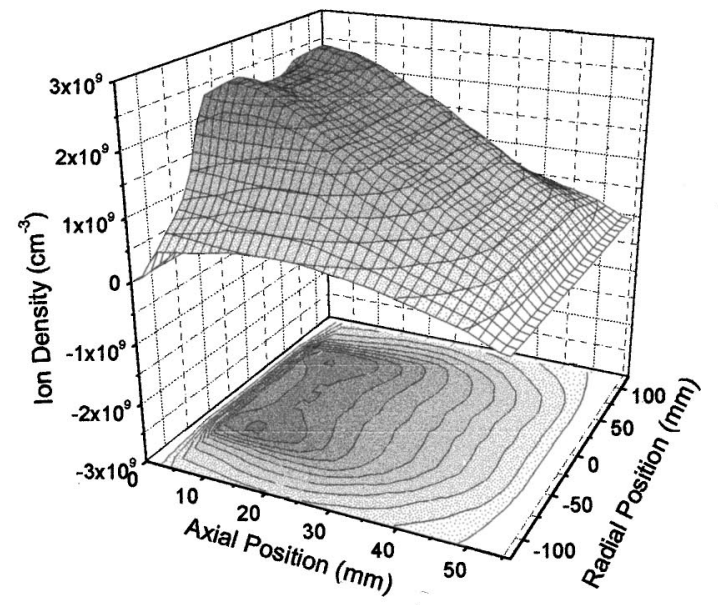

(c)

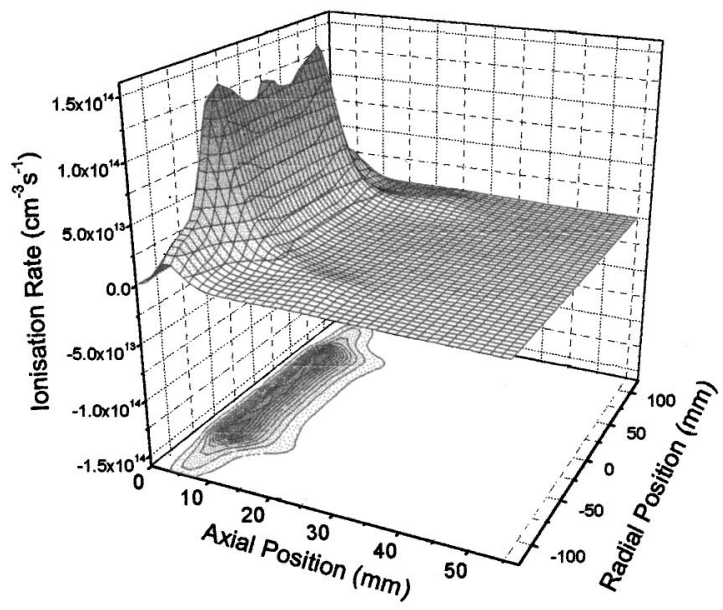

FIG. 1. (a) Two-dimensional experimental ion density profile for an argon discharge at 300 mTorr, $5 \mathrm{~W}$ of rf power. (b) The corresponding modeled ion density profile, and (c) the ionization distribution that results in the density profile in (b)

The collision frequency $\nu_{i z}$ is

$$
\nu_{i z}=n_{g} K_{i z}=n_{g}\langle\sigma(v) v\rangle_{v},
$$

where $m$ and $T_{e}$ are the electron mass and temperature and $v$ is the electron velocity. The particle generation rate, $G$ is considered to be entirely due to ionization by electronneutral collisions

$$
G=\nu_{i z} n_{e} .
$$

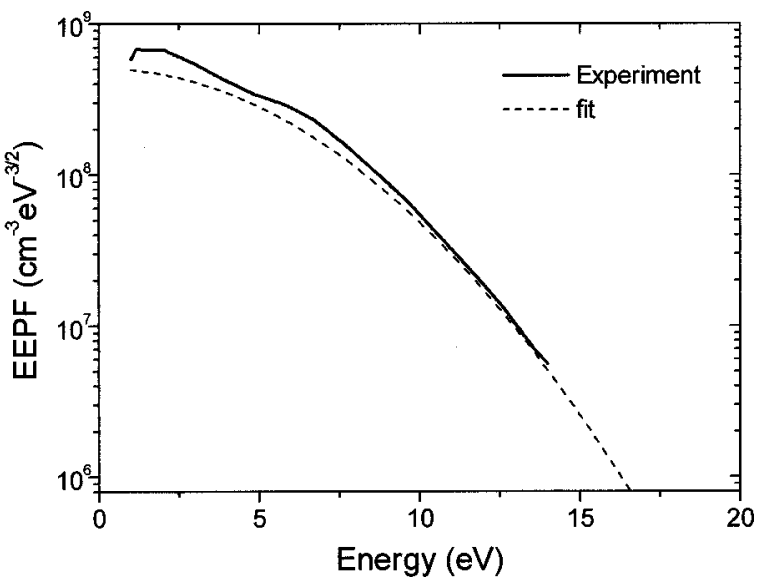

FIG. 2. Measured EEPF plus analytical fit for an argon discharge at 300 mTorr, $5 \mathrm{~W}$ of $\mathrm{rf}$ power.

Figure 2 shows an EEPF measured in the midplane of the 300 mTorr, $5 \mathrm{~W}$ discharge. A Druyvesteyn curve is fit to this data as shown. The curve is preferentially fit to the "tail" of the EEPF as this is most relevant to the ionization. This fit is normalized, multiplied by the ionization cross section, and then multiplied by the electron velocity. The result is then numerically integrated to give the rate constant, as it is in Eq. (5) for a Maxwellian distribution.

This method of calculating the ionization rate is largely dependent on the method used to calculate the EEPF. In this instance, the choice of the plasma potential (zero on the EEPF) is subject to error. To investigate the effect of this, the plasma potential was shifted by $\pm 0.5 \mathrm{~V}$ and the ionization rate recalculated.

Figure 3 shows the central axial ionization distribution (continuous curve) for a 300 mTorr, $5 \mathrm{~W}$ discharge, calculated using the inversion technique. The points plotted are the generation rates obtained using the integration procedure and Eq. (7). The error bars on the points reflect the variation in calculated ionization rate due to shifting the plasma potential. Excellent agreement between the two methods is achieved. As the integral method only accounts for (electron impact) ground state ionization only, the implication here is that other ionization processes are not significant. This is what would be expected under these experimental condi-

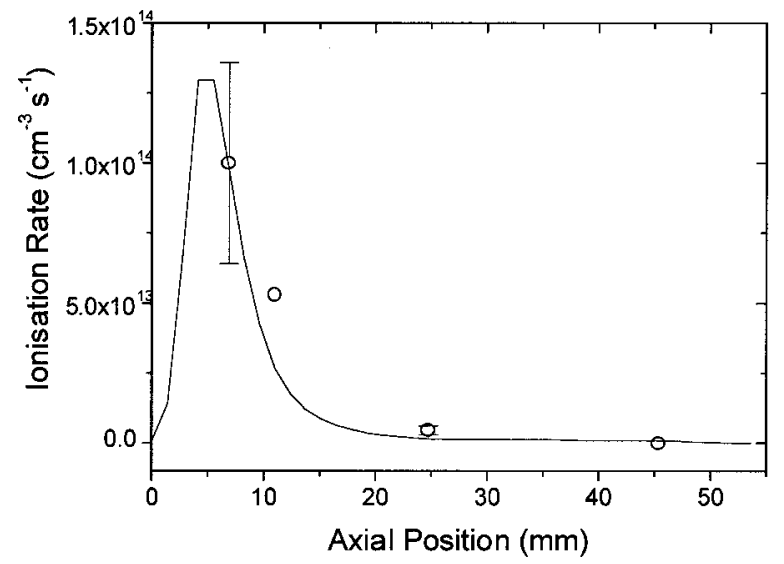

FIG. 3. Axial ionization rate generated using the model approach (solid line) and using the integration method (points). 
tions. A full set of experiments using this technique will be presented in a forthcoming article.

\section{DISCUSSION}

This article has described a method of calculating the ionization rate in two dimensions using the particle density and the diffusion coefficient only. This approach does not require knowledge of the ionization cross sections or the EEPF. Calculation of the rate coefficient using the EEPF can also be limited by the fact that it provides information on electron-neutral ionization only.

This technique has been tested using model source data. The inversion process was observed to reproduce the original source term, thereby verifying that the iteration process converges to a real solution. Hence, there is now a method of determining the ionization rate that is independent of EEPF measurement and that includes all ionization processes, not just ground state ionization by electron-neutral collisions.
Use of the two techniques in tandem to determine the relative contributions of different processes (such as photoionization and two-step ionization) is now a distinct possibility, provided the relevant cross sections and energy distribution functions are known.

${ }^{1}$ J. Coburn, IEEE Trans. Plasma Sci. 19, 1048 (1991).

${ }^{2}$ J. Shohet, IEEE Trans. Plasma Sci. 19, 725 (1991).

${ }^{3}$ D. Graves, IEEE Trans. Plasma Sci. 22, 31 (1994).

${ }^{4}$ T. Kitajima, M. Izawa, N. Nakano, and T. Makabe, J. Phys. D 30, 1783 (1997).

${ }^{5}$ M. B. Hopkins and W. G. Graham, Rev. Sci. Instrum. 57, 2210 (1986).

${ }^{6}$ C. M. Deegan, J. P. Goss, D. Vender, and M. Hopkins, Appl. Phys. Lett. 74, 1969 (1999)

${ }^{7}$ S. C. Brown, Basic Data of Plasma Physics (AIP Press, New York, 1994).

${ }^{8}$ P. A. Jansson, Deconvolution (Academic, New York, 1984).

${ }^{9}$ G. T. Herman, Image Reconstruction from Projections (Academic, New York, 1980).

${ }^{10}$ M. Lieberman and A. Lichtenberg, Principles of Plasma Discharges and Materials Processing (Wiley, New York, 1994). 